\title{
Época de colheita e qualidade pós-colheita do caqui cv. Fuyu com a aplicação pré-colheita de ácido giberélico e aminoetoxivinilglicina
}

\author{
Julius Blum ${ }^{1}$ \\ Ricardo Antonio Ayub ${ }^{2 *}$ \\ Marcelo Barbosa Malgarim ${ }^{3}$ \\ ${ }^{1}$ PPG em Agronomia, Universidade Estadual de Ponta Grossa (UEPG) \\ julisblum@yahoo.com.br \\ ${ }^{2}$ Departamento de Fitotecnia e Fitossanidade, UEPG \\ Av. Carlos Cavalcante, 4748, CEP 84030-900, Ponta Grossa - PR, Brasil \\ rayub@uepg.br \\ ${ }^{3}$ Instituto Agronômico do Paraná (IAPAR) \\ marcelo_malgarim@iapar.br \\ *Autor para correspondência
}

Submetido em 27/04/2008

Aceito para publicação em 05/08/2008

\section{Resumo}

O objetivo do presente trabalho foi avaliar o efeito do ácido giberélico $\left(\mathrm{AG}_{3}\right)$ e de aminoetoxivinilglicina (AVG), aplicados em pré-colheita, visando retardar a colheita e a qualidade pós-colheita de caquis cv. Fuyu para aumentar o período de oferta de frutos. $\mathrm{O}$ experimento foi realizado em blocos ao acaso e os tratamentos

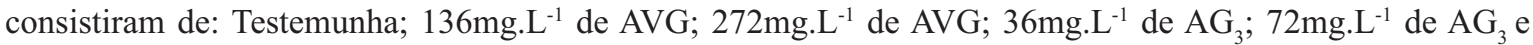

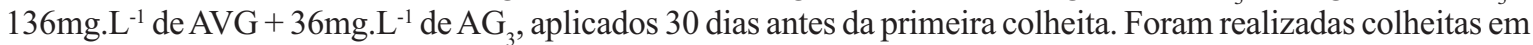
duas épocas e os frutos foram armazenados por 30 dias a $4^{\circ} \mathrm{C}$. As avaliações químicas e físicas dos frutos foram realizadas na colheita e em intervalos de 15 dias seguidos de quatro dias a $20^{\circ} \mathrm{C}$. Concluiu-se que a aplicação de AVG na concentração de $136 \mathrm{mg} . \mathrm{L}^{-1}$ ou $\mathrm{AG}_{3}$ na concentração de $72 \mathrm{mg} . \mathrm{L}^{-1}$ mantiveram a firmeza, prolongando o período de colheita em 20 dias. Os frutos colhidos em estádio inicial de maturação foram mais sensíveis à injúria por frio, e não suportaram 15 dias de armazenagem a $4^{\circ} \mathrm{C}$. Os reguladores vegetais não foram eficientes em prolongar o período pós-colheita, no entanto retardaram o amadurecimento, visto que a concentração de açúcares nos frutos tratados foi menor que nos frutos sem tratamento.

Unitermos: reguladores vegetais, Diospyrus kaki, manejo

\section{Abstract}

Harvest time and post-harvest quality of Fuyu persimmon treated before harvest with gibberellic acid and aminoetoxyvinilglycine. The aim of this work was to evaluate the effects of gibberellic acid (GA $)$ and aminoetoxyvinilglycine (AVG) applied in preharvest spraying, on the retardation of the harvest and on the quality of persimmon fruits cv. Fuyu. The experiment was carried in randomized complete block design. The treatments were: control, $136 \mathrm{mgL}^{-1}$ of AVG, $272 \mathrm{mgL}^{-1}$ of AVG, $36 \mathrm{mgL}^{-1}$ of GA $72 \mathrm{mgL}^{-1} \mathrm{of} \mathrm{GA}_{3}$ and 136 $\mathrm{mgL}^{-1}$ of $\mathrm{AVG}+36 \mathrm{mgL}^{-1}$ of $\mathrm{GA}_{3}$, spraying 30 days before the first harvest. The fruits were harvested twice 
and stored at $4^{\circ} \mathrm{C}$. The chemical and physical evaluations of the fruits were carried out the date of the harvest and at intervals of 15 days followed by four days at $20^{\circ} \mathrm{C}$. In conclusion, the application of AVG $\left(136 \mathrm{mgL}^{-1}\right)$ or $\mathrm{GA}_{3}\left(72 \mathrm{mgL}^{-1}\right)$ maintained the firmness of the fruits and delayed harvest by twenty days. However, fruits harvested in the initial state of ripening were more sensitive to chilling injury and were unable to support 15 days of storage at $4^{\circ} \mathrm{C}$. The plant growth regulators were not efficient in prolonged storage. However, ripening was delayed due to the fact that the concentration of sugars was lower in the treatments than in the control.

Key words: plant growth regulator, Diospyrus kaki, crop science

\section{Introdução}

O caqui (Diospyrus kaki, L.) é um fruto subtropical cuja cultura vem despertando grande interesse, devido aos elevados rendimentos que proporciona aos produtores (Sarria, 1998). O Brasil possui 8.322ha plantados com caqui com uma produção de 164.849ton e produtividade de $19.839 \mathrm{~kg} / \mathrm{ha}$ (Reetz, 2007). No entanto, a produção brasileira se concentra na cv. Fuyu, que tem sua colheita nos meses de março e abril. O curto período de colheita e o baixo tempo de conservação pós-colheita (Fagundes et al., 2006) causam um excesso de oferta no período de safra, ocorrendo queda do preço do produto.

A maturação é concentrada em um curto período de tempo e pode levar a perda de frutos no início da colheita, principalmente em condições adversas de campo. Esses eventos são regulados pelo etileno (Masia et al., 1998) via aumento de atividade da celulase e da poligalacturonase na zona de abscisão, os quais podem causar a queda prematura do fruto (Ward et al., 1999). Guis et al. (1997) trabalhando com melões transgênicos com inibição da síntese de etileno evidenciaram o papel de vias dependentes e independentes deste hormônio na maturação, fato que posteriormente foi confirmado por Pech et al. (2003).

Para ampliar o período de colheita e prolongar a oferta de frutos, necessita-se estabelecer novas condições de manejo na pré-colheita, colheita e armazenamento, tais como o emprego de reguladores vegetais, os quais podem ser utilizados para retardar a maturação e preservar a qualidade pós-colheita do fruto.

Aminoetoxivinilglicina (AVG) é um inibidor da síntese de etileno, extraído de culturas de Streptomyces $s p$. (Latche et al., 1995), e tem apresentado bons resultados na prevenção da queda prematura de frutos de pessegueiro (Amarante et al., 2002), aumento da firmeza e retardo da colheita de maçã (Dabul e Ayub, 2005). Aplicações pós-colheita de AVG em caqui Fuyu mantiveram a firmeza dos frutos por 20 dias a temperatura ambiente (Gonzalez et al., 2004) e refrigerado (Fagundes et al., 2006).

Tratamentos pré-colheita, como a aplicação de ácido giberélico, podem retardar a maturação dos caquis. O efeito do ácido giberélico parece estar relacionado com a redução na velocidade de evolução na coloração das frutas, retardando a colheita, por meio da inibição da ação das clorofilases, que resulta em retardamento da degradação das clorofilas e no acúmulo de carotenóides (Danieli et al., 2002). Além disso, promove maior retenção da firmeza da polpa e aumenta sua conservação pós-colheita em temperatura ambiente por até 15 dias (Ben-Airie et al., 1986). Frutas tratadas com $\mathrm{AG}_{3}$ são menos suscetíveis ao aparecimento de escurecimento da casca (Ferri et al., 2002a). Uma mistura de AVG 50ppm e $\mathrm{AG}_{3} 30 \mathrm{ppm}$, aplicada 30 dias antes da colheita, mostrouse eficiente em prolongar até 16 dias a conservação do caqui 'Fuyu' (Ferri et al., 2002b) e de pêssegos cv. Rubidoux (Amarante et al., 2005).

O objetivo do presente trabalho foi avaliar o efeito do ácido giberélico e de aminoetoxivinilglicina, aplicados em pré-colheita, no retardo da colheita e na qualidade pós-colheita, em câmara fria, de caquis cv. Fuyu.

\section{Material e Métodos}

O experimento foi realizado em plantas de caqui cv. Fuyu pertencentes a um pomar comercial localizado em Porto Amazonas, PR. Os tratamentos foram aplicados no dia 17 de fevereiro de 2007 e foram realizadas duas colheitas, no dia 20 de março no início do amarelecimento dos frutos e 10 de abril coletando- 
se os frutos já de coloração amarela, remanescentes nas plantas, num total de 20 frutos por parcela, em cada colheita. Os frutos foram transportados imediatamente após a colheita para análise e armazenamento.

Os tratamentos foram: Testemunha (água); $136 \mathrm{mgL}^{-1}$ de AVG; $272 \mathrm{mgL}^{-1} \mathrm{de}$ AVG; $36 \mathrm{mgL}^{-1} \mathrm{de}$ $\mathrm{AG}_{3} ; 72 \mathrm{mgL}^{-1}$ de $\mathrm{AG}_{3}$ e $136 \mathrm{mgL}^{-1}$ de $\mathrm{AVG}+36 \mathrm{mgL}^{-1}$ de $\mathrm{AG}_{3}$, sendo pulverizados 2 litros por planta até o ponto de escorrimento. Os frutos foram armazenados a temperatura de $4^{\circ} \mathrm{C} \pm 1^{\circ} \mathrm{C}$, e UR de $80 \%$. As avaliações foram quinzenais seguidas de quatro dias à temperatura de $20 \pm 1^{\circ} \mathrm{C}$, simulando o período de comercialização.

Os parâmetros avaliados foram: firmeza de polpa (N) com penetrômetro com ponteira de $8 \mathrm{~mm}$ em dois pontos da zona equatorial dos frutos; sólidos solúveis determinados por refratometria em ${ }^{\circ}$ Brix; acidez por titulação com $\mathrm{NaOH}$ e resultado expresso em \% de ácido málico; $\mathrm{pH}$ do suco puro; teor de pectina da fruta pelo método de McCready e McComb (1952) e perda de massa fresca na câmara fria e a $20^{\circ} \mathrm{C}$.

$\mathrm{O}$ experimento foi conduzido em blocos ao acaso com seis tratamentos e quatro repetições de cinco plantas. Em cada colheita foram coletados 20 frutos por parcela, as avaliações foram realizadas em grupos de quatro frutos em cada tempo de armazenamento. A comparação dos frutos entre épocas de colheita foi em esquema fatorial 6 (tratamentos) x 2 (épocas de colheita), e o efeito do tempo de conservação da segunda colheita foi avaliado em esquema fatorial 6 (tratamentos) x 3 (períodos de armazenamento), já que os frutos perderam a qualidade de comercialização aos trinta dias. As médias foram comparadas pelo teste de Tukey $(p \leq 0,05)$.

\section{Resultados e Discussão}

Não ocorreu interação significativa entre tratamentos e época de colheita dos frutos para as variáveis avaliadas, desta forma os resultados foram avaliados independentemente.

Os tratamentos não afetaram significativamente a massa de frutos, acidez titulável, $\mathrm{pH}$ do suco e teor de sólidos solúveis (Tabela 1). Entretanto a firmeza foi superior nos tratamentos com $\mathrm{AG}_{3}$ na maior dosagem e
AVG na menor concentração em relação à testemunha, concordando com os resultados obtidos por Danieli et al. (2002) e Ferri et al. (2002b), o que demonstra a eficiência destes tratamentos.

TABELA 1: Valores médios de massa fresca, $\mathrm{pH}$, acidez titulável, sólidos solúveis e firmeza de frutos de caqui 'Fuyu' tratados com AVG e $\mathrm{AG}_{3}$. Ponta Grossa, PR, 2007.

\begin{tabular}{l|c|c|c|c|c}
\hline \multirow{2}{*}{ Tratamentos } & $\begin{array}{c}\text { Massa } \\
\text { fresca } \\
\text { g.fruto }\end{array}$ & pH & AT & SS & Firmeza \\
\hline Testemunha & 272 & 6,03 & 0,040 & 13,46 & $59,9 \mathrm{~b}$ \\
\hline $136 \mathrm{mg} \cdot \mathrm{L}^{-1} \mathrm{AVG}$ & 261 & 5,98 & 0,042 & 13,01 & $66,1 \mathrm{a}$ \\
\hline $272 \mathrm{mg} \cdot \mathrm{L}^{-1} \mathrm{AVG}$ & 244 & 6,01 & 0,040 & 13,28 & $62,8 \mathrm{ab}$ \\
\hline $36 \mathrm{mg} \cdot \mathrm{L}^{-1} \mathrm{AG}_{3}$ & 264 & 6,02 & 0,042 & 13,01 & $61,6 \mathrm{ab}$ \\
\hline $72 \mathrm{mg} \cdot \mathrm{L}^{-1} \mathrm{AG}_{3}$ & 266 & 6,03 & 0,037 & 12,39 & $66.9 \mathrm{a}$ \\
\hline $136 \mathrm{AVG}+36 \mathrm{AG}_{3}$ & 253 & 5,97 & 0,039 & 13,20 & $64,7 \mathrm{ab}$ \\
\hline
\end{tabular}

Médias seguidas da mesma letra não diferem entre si pelo teste de Tukey a $5 \%$ de probabilidade.

Comparando-se os frutos entre épocas de colheita foi verificado que os frutos colhidos precocemente tiveram massa fresca maior (Tabela 2). Por ocasião da primeira colheita, foram selecionados os frutos com amadurecimento mais avançado e os frutos colhidos na segunda época foram os remanescentes nas plantas. É possível concluir que os frutos de maior vigor iniciam o processo de maturação precocemente. Embora em maçã, Dabul e Ayub (2005) obtiveram um ganho de massa nos frutos da segunda colheita tratados com AVG. O menor $\mathrm{pH}$ sem a alteração da acidez titulável parece evidenciar um atraso na maturação que também se confirma pelo menor valor de sólidos solúveis e pela manutenção da firmeza mesmo após 20 dias de retardo na colheita (Tabela 2).

TABELA 2: Comparação entre as colheitas em 20/03 e 10/4/07 para a massa fresca, $\mathrm{pH}$, acidez titulável, sólido solúvel e firmeza de frutos de caqui 'Fuyu', tratados com $\mathrm{AVG}$ e $\mathrm{AG}_{3}$. Ponta Grossa, PR, 2007.

\begin{tabular}{c|c|c|c|c|c}
\hline $\begin{array}{c}\text { Época de } \\
\text { colheita }\end{array}$ & Massa fresca & pH & AT & SS & Firmeza \\
\hline $20 / 03 / 07$ & g & & \% ac. málico & ${ }^{\circ}$ Brix & N \\
\hline $10 / 04 / 07$ & $221,25 \mathrm{a}$ & $6,09 \mathrm{a}$ & $0,040 \mathrm{a}$ & $13,32 \mathrm{a}$ & $64,25 \mathrm{a}$ \\
\hline
\end{tabular}

Médias seguidas da mesma letra não diferem entre si pelo teste de Tukey a $5 \%$ de probabilidade. 
Os frutos da primeira colheita, armazenados em câmara fria, sofreram injúria aos 15 dias, com conseqüente perda de firmeza e qualidade para comercialização. Deste modo a avaliação dos tratamentos nos caquis armazenados não pôde ser realizada.

No estudo do armazenamento dos frutos da segunda colheita os tratamentos com $\mathrm{AVG}$ e $\mathrm{AG}_{3}$ não provocaram alterações na perda de massa fresca, teor de pectina, acidez titulável e firmeza dos frutos durante o período armazenado (Tabela 3). Ocorreu maior teor de sólidos solúveis para a testemunha, indicando o avanço na maturação em relação aos demais tratamentos atingindo 14 ${ }^{\circ}$ Brix (Tabela 3). O maior acúmulo de açúcares no tratamento testemunha também foi relatado por Danieli et al. (2002), Gonzalez et al. (2004) e Fagundes e Ayub (2005).

TABELA 3: Valores médios de três tempos de avaliações, de perda de massa fresca, firmeza, $\mathrm{pH}$, acidez titulável, sólido solúvel e teor de pectina de frutos de caqui 'Fuyu', tratados com $\mathrm{AVG}_{\text {e }} \mathrm{AG}_{3}$. Ponta Grossa, PR, 2007.

\begin{tabular}{l|c|c|c|c|c|c}
\hline \multirow{2}{*}{ Tratamento } & $\mathbf{P M}^{*}$ & Firmeza & $\mathbf{p H}$ & AT & SS & Pectina \\
\cline { 2 - 7 } & $\mathbf{g} / \mathbf{1 0 0 g}$ & $\mathbf{N}$ & & $\mathbf{\%} \mathbf{a c .} *$ & ${ }^{\mathbf{0}}$ Brix & $\mathbf{\%}$ \\
\hline Testemunha & 3,0 & 31,8 & $5,81 \mathrm{ab}$ & 0,048 & $14,3 \mathrm{a}$ & 0,27 \\
\hline 136ppm AVG & 2,5 & 37,7 & $5,67 \mathrm{~b}$ & 0,050 & $13,5 \mathrm{ab}$ & 0,37 \\
\hline $272 \mathrm{ppm} \mathrm{AVG}$ & 2,5 & 34,5 & $5,78 \mathrm{ab}$ & 0,045 & $13,5 \mathrm{ab}$ & 0,34 \\
\hline $40 \mathrm{ppm} \mathrm{AG}$ & 2,7 & 34,3 & $5,80 \mathrm{ab}$ & 0,046 & $13,6 \mathrm{ab}$ & 0,37 \\
\hline $72 \mathrm{ppm} \mathrm{AG}_{3}$ & 3,0 & 34,3 & $5,78 \mathrm{ab}$ & 0,044 & $13,0 \mathrm{~b}$ & 0,36 \\
\hline $136 \mathrm{AVG}^{+}$ & 2,7 & 31,6 & $5,84 \mathrm{a}$ & 0,043 & $14,0 \mathrm{ab}$ & 0,36 \\
\hline $40 \mathrm{AG}_{3}$ & & & & &
\end{tabular}

Médias seguidas da mesma letra não diferem entre si pelo teste de Tukey a $5 \%$ de probabilidade. $*$ perda de massa fresca; $* * \%$ de ácido málico.

A acidez manteve-se constante e o $\mathrm{pH}$ variou pouco (Tabela 3), mantendo os valores semelhantes aos obtidos por Fagundes e Ayub (2005) aos 32 dias.

Com o passar do tempo de armazenamento ocorreram alterações em todas as variáveis analisadas. Houve um decréscimo da firmeza, acompanhado pela redução do teor de pectina, inviabilizando a comercialização dos frutos aos 30 dias (Tabela 4). Como resultado da degradação da pectina há uma elevação da acidez titulável e conseqüentemente redução do $\mathrm{pH}$.
Os sólidos solúveis aumentam gradativamente com a maturação ao passo que a perda de massa aumenta com o tempo de armazenagem, tanto no período frigorificado, quanto no tempo de prateleira (Tabela 4).

TABELA 4: Valores médios de perda de massa fresca na câmara fria e após 4 dias a temperatura ambiente, firmeza, $\mathrm{pH}$, acidez titulável, sólido solúvel e teor de pectina de frutos de caqui 'Fuyu', tratados com AVG e $\mathrm{AG}_{3}$, durante o período de armazenamento. Ponta Grossa, PR, 2007.

\begin{tabular}{l|c|c|c|c|c|c|c}
\hline \multirow{2}{*}{ Dias } & PM1 & PM2 & Firmeza & pH & AT & SS & Pectina \\
\cline { 2 - 8 } & $\%$ & $\%$ & $\mathbf{N}$ & & $\begin{array}{c}\text { \% ac. } \\
\text { málico }\end{array}$ & ${ }^{\circ}$ Brix & $\%$ \\
\hline 0 & & & $63,1 \mathrm{a}$ & $5,92 \mathrm{a}$ & $0,040 \mathrm{~b}$ & $12,8 \mathrm{~b}$ & $0,49 \mathrm{a}$ \\
\hline 15 & $1,7 \mathrm{~b}$ & $1,9 \mathrm{~b}$ & $38,3 \mathrm{~b}$ & $5,84 \mathrm{a}$ & $0,034 \mathrm{c}$ & $13,8 \mathrm{a}$ & $0,29 \mathrm{~b}$ \\
\hline 30 & $3,7 \mathrm{a}$ & $2,4 \mathrm{a}$ & $0,71 \mathrm{c}$ & $5,56 \mathrm{~b}$ & $0,063 \mathrm{a}$ & $14,4 \mathrm{a}$ & $0,25 \mathrm{~b}$ \\
\hline
\end{tabular}

Médias seguidas da mesma letra não diferem entre si pelo teste de Tukey a $5 \%$ de probabilidade. PM1 - perda de massa durante o armazenamento refrigerado; PM2 - perda de massa durante o período em temperatura ambiente.

A partir dos resultados, foi possível concluir que:

- A aplicação de $136 \mathrm{mgL}^{-1}$ de AVG ou $72 \mathrm{mgL}^{-1}$ de $\mathrm{AG}_{3}$ manteve a firmeza dos frutos em ambas as colheitas realizadas, prolongando a colheita em 20 dias.

- Os frutos colhidos em estádio inicial de maturação foram mais sensíveis a injúria por frio, não suportando 15 dias de armazenagem a $4^{\circ} \mathrm{C}$.

- Os tratamentos não foram eficientes em manter a firmeza dos frutos durante o armazenamento, porém retardaram o amadurecimento, visto o menor teor de sólidos solúveis dos mesmos.

\section{Referências}

Amarante, C. V. T.; Drehmer, A. M. F.; Souza, F. de; Franscescatto, P. 2005. A pulverização pré-colheita com ácido giberélico $\left(\mathrm{GA}_{3}\right)$ e aminoetoxivinilglicina (AVG) retarda a maturação e reduz as perdas na cultura do pessegueiro. Revista Brasileira de Fruticultura, 27 (1): 1-5.

Amarante, C. V. T.; Simioni, A.; Megguer, C. A.; Blum, L. E. B. 2002. Effect of aminoethoxyvinilglycine (AVG) on preharvest fruit drop and maturity of apples. Revista Brasileira de Fruticultura, 24 (3): 661-664. 
Ben-Airie, R.; Bazak, H.; Blumenfeld, A. 1986. Gibberellin delays harvest and prolongs life of persimmon fruits. Acta Horticulturae, 179: $807-813$.

Dabul, A. N. G.; Ayub, R. A. 2005. Retardo da colheita de maçã (Malus domestica) cv. Gala com a aplicação de Retain ${ }^{\mathrm{TM}}$. Revista Ceres, 52 (302): 1-9.

Danieli, R.; Girardi, C. L.; Parussolo, A.; Ferri, V. C.; Rombaldi, C. V. 2002. Aplicação do ácido giberélico e cloreto de cálcio no retardamento da colheita e na conservabilidade de caqui, Fuyu. Revista Brasileira de Fruticultura, 24 (1): 44-48.

Fagundes, A. F.; Ayub, R. A. 2005. Caracterização físico-química de caquis cv. Fuyu submetidos à aplicação de agentes inibidores de escurecimento e armazenados a $0^{\circ} \mathrm{C}$. Acta Scientiarum, 27 (3): 403-408.

Fagundes, A. F.; Dabul, A. N. G.; Ayub, R. A. 2006. Aminoethoxivinilglicina no controle do amadurecimento de frutos de caqui $\mathrm{cv}$. Fuyu. Revista Brasileira de Fruticultura, 28 (1): 73-75.

Ferri, V. C.; Rinaldi, M. M.; Danieli, R.; Luchetta, L.; Rombaldi, C. V. 2002a. Qualidade de caquis fuyu tratados com cálcio em précolheita e armazenados sob atmosfera modificada. Revista Brasileira Fruticultura, 24 (2): 385-388.

Ferri, V. C.; Rinaldi, M. M.; Danieli, R.; Luchetta, L.; Rombaldi, C. V. 2002b. Controle da maturação de caquis 'Fuyu' com uso de aminoethoxivinilglicina e ácido giberélico. Revista Brasileira de Fruticultura, 24 (2): 344-347.

Gonzalez, A. F.; Ayub, R. A.; Werlang, C. 2004. Efeito de ethephon e embalagem de pve na qualidade pós-colheita de caqui (Diospyros kaki L.) cv. Fuyu armazenados a $25^{\circ} \mathrm{C}$. Publicatio, 10 (1): 21-26.
Guis, M.; Botondi, R.; Ben Amor, M.; Ayub, R.; Bouzayen, M.; Pech, J. C.; Latche, A. 1997. Ripening-associated biochemical traits of Cantaloupe Charentais melons expressing an antisense ACC Oxidase transgene. Journal American Society of Horticultural Science, 122 (6): 748-751.

Latche, A.; Ayub, R.; Martinez, G.; Guis, M.; Ben Amor, M.; Rombaldi, C.; Pech, J. C.; Bouzayen, M. 1995. Biosynthèse et mode d'action de l'hormone végétale éthylène. Fruits, 50: 379-396.

Masia, A.; Ventura, M.; Gemma, H.; Sansavini, S. 1998. Effect of some plant growth regulator tretments on apple fruit ripening. Plant Growth Regulation, 25 (2): 127-134.

McCready, R. M.; McComb, E. A. 1952. Extraction and determination of total pectic materials in fruits. Analyctical Chemistry, 24 (12): 1586-1588,

Pech, J. C.; Ben Amor, M.; Zhengguo, L.; Fikri, El Y.; Ayub, R. A.; Romojaro, F.; Flores, F.; Bernadac, A.; Bouzayen, M.; Latche, A. 2003. Biotechnology of fruit ripening. Down-regulation of ACC Oxidase gene expression in the melon. In: Nath, P.; Mattoo, A. K.; Ranade, S. A. \& Weil, J. H. (Eds). Molecular Insight in Plant Biology. Ed. Science Publishers, INC, Enfield, Plymouth, USA, UK, p.151-157.

Reetz, E. R. 2007. Anuário Brasileiro da Fruticultura. Ed. Gazeta Santa Cruz, Santa Cruz do Sul, Brasil, 136pp.

Sarria, S. D. 1998. Comportamento pós-colheita de caqui (Diospyros kaki): Avaliação física e química. Dissertação de Mestrado, Universidade Estadual de Campinas, Brasil, 72pp.

Ward, D. L.; Beers, E. P.; Byers, R.E.; Marini, R. P. 1999. Cutting apple fruits induces cellulase activity in the abscission zone. HortScience, 34 (4): 601-603. 